\title{
EDITORIAL
}

\section{What to expect from $\mathrm{Al}$ in oncology}

\author{
An increasing number of studies suggest that artificial intelligence could revolutionize medicine. \\ In oncology, we are only beginning to fully understand the practical implications.
}

In the past few years, the terms 'artificial intelligence' (AI) and 'machine learning' (ML) have become common in the news; several important medical advances have been made using these approaches. Some might conclude that we are witnessing a new era in medicine, although others could be confused. What are AI and $\mathrm{ML}$, and how can they affect the practice of medicine? In oncology, the obvious question is how will AI improve the outcomes of patients with cancer? Summaries of studies addressing these questions have already been covered in Nature Reviews Clinical Oncology, and will continue to receive the attention they deserve.

In the current issue, Anant Madabhushi and co-authors ${ }^{1}$ provide an overview of studies in which AI-based approaches have been applied by pathologists analysing potentially neoplastic tissues. In this Perspective, we learn that researchers have trained computers to distinguish patterns in digitized medical images. Encouraging results have been obtained when the analytical performance of these ML-based approaches is compared with that of expert pathologists. These results alone, however, should not be interpreted as a justification for outsourcing the work of pathologists but rather, as an indication that their workload could be optimized and, importantly, the waiting time for patients to receive a diagnosis can be reduced. Madabhushi et al. also define terms commonly used in AI studies and, thus, we hope that this article will be particularly useful for readers who are less familiar with this field.

Pathology is not the only area in which ML has the potential to improve the outcomes of patients with cancer. Any piece of information that can be translated into patterns, predictable outcomes or pair associations, to mention only a few examples, can be virtually taught to machines. Indeed, AI-based approaches are being used in areas such as radiology and clinical trial design. Another promising use of AI would be the integration of multi-omics data from each individual in order to facilitate the administration of tailored treatments.

In their article, Madabhushi et al. acknowledge the current challenges to implementing approaches using $\mathrm{AI}$ in routine clinical settings. Defining standards is one of these challenges: situations exist in which the same clinical question has been addressed in independent institutions with the development of separate ML tools, each one validated in a particular set of samples.
Clinicians worldwide need to have the assurance that they can rely on any of those ML approaches when they encounter a similar clinical scenario in their own practice. Collaboration among regulatory bodies, technology developers and clinical staff will be key to define high-quality standards for the use of $\mathrm{AI}$ in all relevant areas of clinical oncology.

Another challenge that has already received attention is that of access to care. Eventually, individuals receiving health care at institutions with AI expertise could have better outcomes than those seen in conventional facilities (for example, owing to more timely diagnosis and real-time disease monitoring). Some experts believe that, similarly to other new technologies, the costs associated with using AI will only be high during an initial period and will decrease over time. This expectation is not unreasonable, nonetheless, many medical centres will not be able to afford the initial investment of resources to introduce these tools in their practice. Let's not forget that, in order to implement AI-based tools, institutions will need careful financial planning, but also a critical mass of medical professionals trained in this new approach to medicine. Thus, widespread access to AI-based health care might not happen in the near future.

Finally, some experts are optimistic and believe that, with access to AI, clinicians will have extra time to interact with their patients. Such a shift will only take place if the total duration of patient visits remains the same and $\mathrm{AI}$ is not used instead to cover for staff shortages. Importantly, some patients might perceive the adoption of AI by their clinicians as a disruptive element communication will be key to help them understand the different roles of the human and the machine in their care.

In summary, the practical implications of using AI in routine oncology practice are not yet completely understood. In addition to the challenges discussed, prospective evidence of the potential benefits of using AI in medicine remains limited, thus necessitating further research. The introduction of AI into routine clinical practice is a complex effort that will require multidisciplinary expertise and, more importantly, the input of patients and their families and the cooperation of regulatory bodies.

\footnotetext{
Bera, K. et al. Nat. Rev. Clin, Oncol. https://doi.org/10.1038/ s41571-019-0252-y (2019).
} 